\title{
First Potential Reinfection Case with SARS-Cov-2 in Lebanon: A Case Study
}

\author{
Fadi Abdel Sater ${ }^{1 *}$, Kassem Hamze ${ }^{1}$, Iman Younes ${ }^{2}$, Nadia hamade hajj ${ }^{3}$, Fatima Farhat ${ }^{3}$, Paul \\ Nguewa $^{4 *}$ and Mahmoud Younes ${ }^{5}$ \\ ${ }^{1}$ Laboratory of Molecular Biology and Cancer Immunology (Covid 19 Unit), Faculty of sciences I, Lebanese University, Hadath,
} Beirut, Lebanon

${ }^{2}$ Faculty of Medecine, Lebanese University, Lebanon

${ }^{3}$ Safety department, Beirut Cardiac institute, Lebanon

${ }^{4}$ University of Navarra, ISTUN Instituto de Salud Tropical, Department of Microbiology and Parasitology., IdiSNA (Navarra Institute for Health Research). c/ Irunlarrea 1, Spain

${ }^{5}$ Research Department, Beirut Cardiac institute, Lebanon

*Corresponding author: Fadi Abdel Sater, Laboratory of Molecular Biology and Cancer Immunology (Covid 19 Unit), Faculty of sciences I, Lebanese University, Hadath, Beirut, Lebanon

Paul Nguewa, University of Navarra, ISTUN Instituto de Salud Tropical, Department of Microbiology and Parasitology. IdiSNA (Navarra Institute for Health Research). c/ Irunlarrea 1, 31008 Pamplona, Navarra, Spain

\section{ARTICLE INFO}

Received: 幽 February 04, 2021

Published: 慧 February 10, 2021

Citation: Fadi Abdel Sater, Kassem Hamze, Iman Younes, Nadia hamade hajj, Fatima Farhat, Paul Nguewa, Mahmoud Younes. First Potential Reinfection Case with SARSCov-2 in Lebanon: A Case Study. Biomed J Sci \& Tech Res 33(5)-2021. BJSTR. MS.ID.005456.

Keywords: Reinfection; SARS-CoV-2; Immunity; Lebanon; Pandemic

\section{ABSTRACT}

Background: The immune response to severe acute respiratory syndrome coronavirus 2 [SARS-CoV-2] and the degree of protective immunity against the disease or the infection still unknown. So far, researchers are unable to fully understand the possibility of reinfection with SARS-CoV-2. Here, we describe the first possible reinfection by SARS-CoV-2 in Lebanon.

Methods: A 28-year-old woman who is a nurse presented to our health care service in our hospital on 2 occasions. The first was on the 1st of November 2020, when she had mild symptoms of SARS- CoV-2 infection, and the second was on the 24th of December, following an incidence of close contact with a confirmed COVID-19 case at the hospital unit where she is working. Nasopharyngeal swabs were obtained from the patient at each presentation and during follow-up. Rtq PCR testing was done to confirm SARS-CoV-2 infection. IgM and IgG were quantified in serum to monitor the immune response of this case.

Results: The patient had two positive tests for SARS-CoV-2, the first on November first, and the second on December 24, separated by two consecutive negative tests done during follow-up in December. The first infection was symptomatic and the symptoms disappeared 10 days after the test date. The second infection following a contact with confirmed covid19 patient was asymptomatic with a detectable amount of IgG and a short incubation period.

Conclusion: Previous infection with SARS-CoV-2 does not necessarily guarantee a total immunity. It is essential to elucidate the characteristics and frequency of reinfections, as this could impact our understanding immunity acquired after natural infection.

\section{Introduction}

One of the key questions amidst the Covid-19 pandemic is understanding how long immunity may last for people exposed to SARS-CoV-2. As the protective immunity against this virus is not fully understood, more information is needed to fill the gaps in our understanding. Protection against severe disease has been shown in animal models and inferred in humans infected with 
the virus. Deng et al. showed that the primary exposure to SARSCoV-2 protects against reinfection in rhesus macaques [1]. Cases of re-infection are rarely reported which could mean that Immunity is probable, at least in the short term. Several published and confirmed cases of reinfection were reported [2-8]. They showed an interval between episodes from 48 to 185 days. The first case of re-infection of COVID-19 was reported in Hong Kong with second episode of asymptomatic infection. Two studies in USA showed a Genomic evidence of a case report for symptomatic reinfection with SARS-CoV-2 within a period of 48 [2]. and 90 days [4]. Recently, Hanrath et al. observed no symptomatic reinfections in a cohort of healthcare workers [8]. As more cases of reinfection surface may help the scientific community to understand better how frequently natural infections with SARS-CoV-2 induce the level of immunity and the correlates of protection. This information is key to understanding which vaccines are capable of crossing that threshold to confer individual and herd immunity.

\section{Materials and Methods}

\section{Study Case and Clinical Specimens}

We present a case report of 28-year-old female was a nurse at our hospital with no history of clinically significant underlying conditions. She had no sign of compromised immunity. The patient provided written signed informed consent. Samples were collected in Beirut cardiac institute. Nasopharyngeal swab samples from the patient were collected into a sterile container containing VTM.

\section{SARS-CoV-2 rRT-PCR}

Viral RNA from Nasopharyngeal swab were extracted $200 \mu \mathrm{L}$ of VTM was used for RNA purification. RNA was extracted from the clinical samples on Kingfisher flex purification system Thermo Fisher using MagMAX ${ }^{\mathrm{TM}}$ Viral/Pathogen Nucleic Acid Isolation Kit (Thermo fisher). Reactions were performed in in $20 \mu \mathrm{L}$ final volume reaction containing $5 \mu \mathrm{L}$ of extracted RNA, rRT-PCR was performed using QuantStudio 5 real-time PCR detection system (Thermo fisher) and TaqPath 2019-nCoV real-time PCR kit (Thermo fishe), which targeted the RdRP, N and Spike genes of SARS-CoV-2.

\section{Detection of Antibodies Against SARS-CoV-2}

Detection of serum IgG and IgM antibodies against the SARSCoV-2 nucleocapsid and spike protein respectively were carried out in the Architect analyser using Abbott's SARS-CoV-2 IgG and IgM assays (Abbott, Abbott Park, IL, USA) following manufacturer's instructions. The cut- off index value was $\geq 1.4$ for positive IgG and $\geq 1$ for positive IgM.

\section{Results}

On November first, she had the following symptoms: a fever, a cough, muscle pain and sudden loss of smell and taste. After a nasopharyngeal swab, she was tested positive for SARS-CoV-2 via a PCR-test with high viral load. The patient was isolated for three weeks, after which she tested negative twice before returning to work. A serology test taken on the same day showed a detectable amount of the IgG SARS-Cov-2-antibody. 47 days later, one of her colleagues working in the same hospital unit was diagnosed as a COVID 19 patient. A week after contact, she and 6 of 8 of her colleagues were tested positive for the virus. She retested positive on the second day to confirm the infection. This second COVID-19episode was asymptomatic and only lasted a week. Tests taken on the $31^{\text {st }}$ of December showed no detection of the virus by PCR and a detectable amount of IgG (Figure 1).

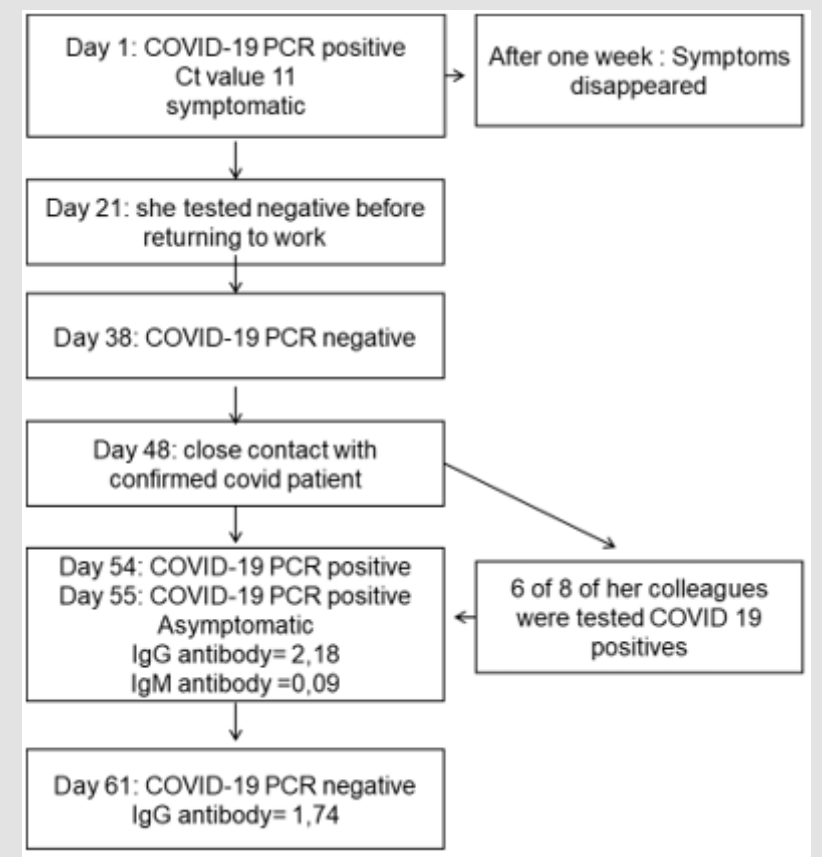

Figure 1: Timeline of severe acute respiratory syndrome coronavirus 2 reinfection over a period of 54 days. 


\section{Discussion}

Recently, several cases of reinfection have been documented worldwide. Our case report presents details of the first individual in Lebanon to have asymptomatic potential reinfection with SARSCoV-2: a female 28-year-old whose first and second positive tests were carried out 54 days apart, with two consecutive negative tests in between. The patient was symptomatic with high viral load in the first episode and asymptomatic in the second one. This has similarities with the reinfection case in Hong Kong that described reinfection in a 33-year-old patient within a period of 142 days [2]. In the USA, a reinfection was described in a 25-year- old male within a period of 48 days. The patient was symptomatic during both episodes, with the second episode being more severe [3]. Another similar case of reinfection was described in the USA in a 42-year-old male, 90 days after the first infection episode [4]. Van Elslande et al. reported reinfection in a 52-year-old female from Belgium, within a period of 93 days with mild symptoms during both episodes [5] Prado-Vivar et al. reported reinfection in a 46-year- old male from Ecuador with symptoms during both episodes while the first and the second test were carried out 63 days apart. This patient had a contact with a confirmed COVID 19 patient before the second infection [6]. Moreover, two young healthcare workers were tested during screening campaigns and were reported as reinfected cases. The episodes were separated by a period of $] 63$ days and were asymptomatic both times [7].

Recently, Hanrath et al. observed no symptomatic reinfections in a cohort of healthcare workers [8]. Most of these cases were established on the basis of comparative whole genome sequencing, and the identification of single nucleotides variations. In our case, we weren't able to perform sequencing of isolate strains due to the lack of funding. While other studies indicate that caution should be exercised when comparing two samples from the same patient. Jary et al. analyzed the genomic diversity of SARS-CoV-2 in a patient with longitudinal follow-up; The authors suggest that when analyzing samples from the same patient at different time points, the same variant may not persist over time and it should keep in mind the heterogeneity of the population [9]. Re-positivity can also be due to prolonged viral shedding, which is common during SARS- CoV-2 infections. Lancman et al., showed in a case report study that Re-positivity is linked to virus reactivation. This study described a patient with B cell acute lymphoblastic leukemia who presented with two separate episodes of COVID-19. Re-infection in this case appeared unlikely, the patient hadn't contact with a COVID-19 confirmed case. In this case, immunosuppressive therapy may result in loss of COVID-19 antibodies which is consistent with reactivation of the virus [10].

Our patient had no immunological disorder that would involve the facilitation of reinfection, nor was she taking any immunosuppressive medication. Furthermore, A Chinese study found that among the COVID-19 cases released, 14\% were retested as positive for SARS-CoV-2 over a period ranging from 2 to 19 days. They showed that there was no link established between re-positive test and weaker immunity in these cases. In addition, a study on 285 re-positive cases, showed that $59.6 \%$ of re-positive cases were detected by screening and $44.7 \%$ were due to symptoms with an average of 44.9 days. $89.5 \%$ of these cases had Ct values $>30$ and all had negative viral cell cultures [11]. In our case, the patient had a high-risk close contact with a confirmed COVID-19 case one week before the second positive result. Six out of eight of her colleagues were infected following this contact. The two positive tests were separated by two negative PCR tests. Furthermore, two independent PCR positive results were obtained in the potential second reinfection with a moderate viral load (CT-value $=27,3$ viral genes). The IgG test showed a moderate increase of the level of IgG. It should be noted that the frequency of reinfection is very rare and cannot be defined by the cases mentioned so far. But if our patient and the other cases mentioned are in fact re-infections, the initial exposure to SARS-CoV-2 may not result in full immunity levels. A major limitation of our case study is that we were unable to do the sequencing and perform an evaluation of the immune response to the first episode of SARS-CoV-2 infection. We were also unable to fully assess the effectiveness of the immune responses during the 2 episodes.

\section{Conclusion}

Previous exposure to SARS-CoV-2 does not necessarily translate into guaranteed full immunity. Our results may have implications for the role of vaccination. In addition, since influenza regularly shows the challenges of effective vaccine design [12], it is important to mobilize people to take precautions to prevent infection with SARS-CoV-2, regardless of whether or not they have already been diagnosed. Further work is essential to study and evaluate immune reactions in vivo vitro after reinfection.

\section{Acknowledgement}

PN thanks Fundación La Caixa (LCF/PR/PR13/11080005), Fundación Caja Navarra, Fundación Roviralta, Ubesol, Inversiones Garcilaso de la Vega, COST Actions CA18217 and CA18218, and EU Project uncover (Grant/Award Number: 101016216) for their support. FAS and KZ thank the Lebanese University its support.

\section{Author Contribution}

FAS and MH conceived of the presented idea, wrote the manuscript with input from all authors. All authors contributed to the design and implementation of the research, to the analysis of the results and discussed the results and contributed to the final manuscript.

\section{Declaration of Competing Interest}

All authors disclose no conflict of interest and no external funding was used for this work. 


\section{References}

1. Deng W, Bao L, Liu J, Xiao C, Liu J, et al. (2020) Primary exposure to SARS-CoV-2 protects against reinfection in rhesus macaques. Science 369(6505): 818-823

2. To KK, Hung IF, Ip JD, Chu AW, Chan WM, et al. (2020) COVID- 19 reinfection by a phylogenetically distinct SARS-coronavirus-2 strain confirmed by whole genome sequencing. Clin Infect Dis 25: ciaa1275.

3. Tillett RL, Sevinsky JR, Hartley PD, Kerwin H, Crawford N, et al. (2021) Genomic evidence for reinfection with SARS-CoV-2: a case study. Lancet Infect Dis 21(1): 52-58

4. Larson D, Brodniak SL, Voegtly LJ, Cer RZ, Glang LA, et al. (2020) A Case of Early Re-infection with SARS-CoV-2. Clin Infect Dis 19: ciaa1436.

5. Van Elslande J, Vermeersch P, Vandervoort K, Wawina Bokalanga T, Vanmechelen B, et al. (2020) Symptomatic SARS-CoV-2 reinfection by a phylogenetically distinct strain. Clin Infect Dis 5: ciaa1330.

6. Prado Vivar B, Becerra Wong M, Guadalupe JJ, Márquez S, Gutierrez B, et al. (2020) A case of SARS-CoV-2 reinfection in Ecuador. Lancet Infect Dis S1473-3099(20)30910-5.

\section{ISSN: 2574-1241}

DOI: $10.26717 /$ BJSTR.2021.33.005456

Fadi Abdel Sater, Paul Nguewa. Biomed J Sci \& Tech Res

(c) (P) This work is licensed under Creative

Submission Link: https://biomedres.us/submit-manuscript.php
7. Gupta V, Bhoyar RC, Jain A, Srivastava S, Upadhayay R, et al. (2020) Asymptomatic reinfection in two healthcare workers from India with genetically distinct SARS-CoV-2. Clin Infect Dis 23:ciaa1451.

8. Hanrath AT, Payne BAI, Duncan CJA (2020) Prior SARS-CoV-2 infection is associated with protection against symptomatic reinfection. J Infect 26: S0163-4453.

9. Jary A, Leducq V, Malet I, Marot S, Klement Frutos E, et al. (2020) Evolution of viral quasispecies during SARS-CoV-2 infection. Clin Microbiol Infect 26(11): 1560-1560.

10. Lancman G, Mascarenhas J, Bar Natan M (2020) Severe COVID-19 virus reactivation following treatment for $\mathrm{B}$ cell acute lymphoblastic leukemia. J Hematol OncolJ Hematol Oncol 13(1): 131.

11. Lu J, Peng J, Xiong Q Liu Z, Lin H, et al. (2020) Clinical, immunological and virological characterization of COVID-19 patients that test repositive for SARS-CoV-2 by RT-PCR. EBioMedicine 59: 102960.

12. Wei CJ, Crank MC, Shiver J, Graham BS, Mascola JR (2020) Nextgeneration influenza vaccines: opportunities and challenges. Nat Rev Drug Discov 19(4): 239-252.

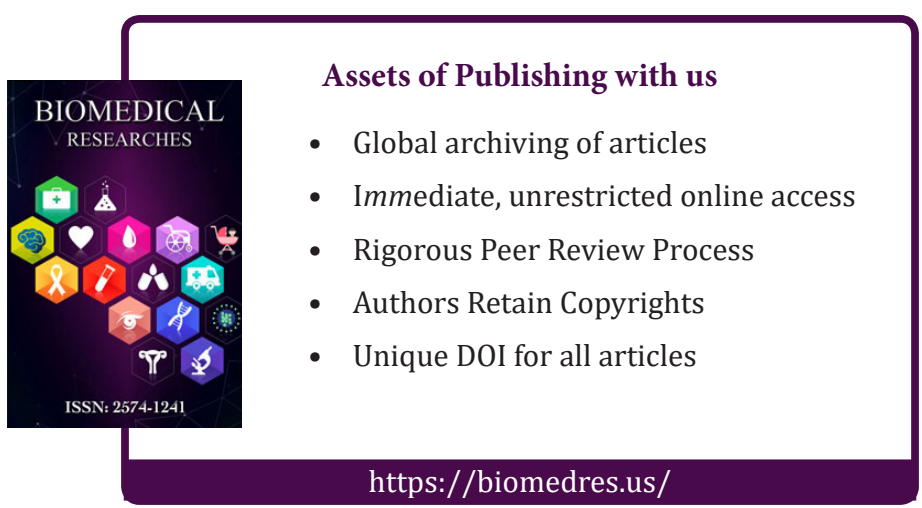

